\title{
Analysis of the cavitating flow induced by an ultrasonic horn- Experimental investigation on the influence of actuation phase, amplitude and geometrical boundary conditions
}

\author{
Saskia Müller ${ }^{1, \mathrm{a}}$, Maurice Fischper ${ }^{1}$, Stephan Mottyll ${ }^{1}$, Romuald Skoda ${ }^{1}$, and Jeanette Hussong ${ }^{1}$ \\ ${ }^{1}$ Ruhr Universität Bochum, Chair of Hydraulic Fluid Machinery, Universitätsstr. 150, 44801 Bochum, Germany
}

\begin{abstract}
Till today, factors influencing the formation and collapse of densely distributed, interacting cavitation bubbles are only qualitatively understood. The aim of the present study is to investigate experimentally the influence of selected boundary conditions on the number and size distribution of cavitation bubbles created by an ultrasonic horn (sonotrode). Cavitation bubble clouds below the sonotrode were recorded by means of phase-locked shadowgraphy imaging. The time integrated number of cavitation bubbles was found to decrease exponentially with growing bubble radius. The number of bubbles was increased with growing actuation amplitude and gap width between the sonotrode tip and an opposing solid wall. Furthermore, it could be shown that the number of cavitation bubbles depends on the actuation phase. Future investigations will focus on establishing a statistical relation between the number and size distribution of cavitation bubbles in the near wall region and the resulting cavitation erosion on solid surfaces.
\end{abstract}

\section{Introduction}

Cavitation is the formation of vapor bubbles which create pressure peaks of several hundred bars during implosion. It is well known that cavitation occurring close to surfaces will cause damage of components. Nevertheless, efforts of minimizing cavitation in hydraulic machinery were only partially successful. One reason for that is the variety of factors influencing cavitation, including the fluid quality such as the content of impurities and dissolved gas [1], but also physical properties of the liquid [2] and surface properties of the liquid-solid interfaces [3].

Recent studies showed that the dissolved gas content is one of the major influencing factors that may provoke cavitation which leads to surface damage [4]. Principle investigations of cavitation-induced material damage are usually performed with an ultrasonic horn, hence on referred to as sonotrode. As already known, bubbles of different sizes move in different directions due to Bjerknes forces [5]. The typical flow field distribution and the cavitation zone are illustrated in Figure 1.

Jayaprakash et al. investigated the influence of ultrasonic cavitation on different materials according to the ASTM G32 [8] norm for tests of "cavitation resistivity" [7]. They analyzed the damage of cavitation by Pitting Tests.

\footnotetext{
${ }^{\mathrm{a}}$ Corresponding author: saskia.s.mueller@rub.de
}

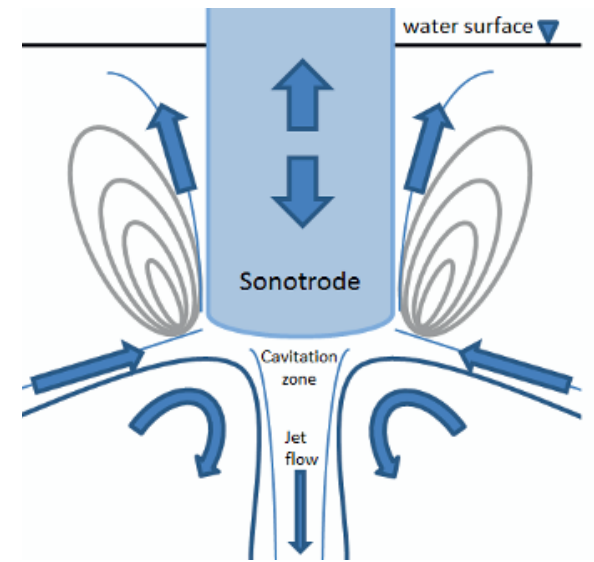

Figure 1. Typical flow field distribution and cavitation zone below the sonotrode. Adapted from Anh [6]

While these studies are purely deterministic, it is only recently that people investigate the underlying two-phase flow to get a better insight in the fluid-surface interaction leading to the material damage. Visualization results from Moussatov et al. [9] revealed the typical cavitation bubble formation and motion that is formed below an ultrasonic horn, which are Cone Bubble Structure (CBS) and Acoustic Lichtenberg Figures (ALF). CBS assume a cone like shape beneath the sonotrode, while the term ALF refers to branched bubble lines. Not only the structure but also the oscillating bubble movement was analyzed by Nowak et al. [10]. They observed jumping, jetting, splitting and repelling of the bubbles by 
producing pseudo streaks. While bubble movement and growth [11] were examined by different people, no attention was paid to the bubble frequency distribution in preceding literature.

Vaidya et al. [12] analyzed the influence of the acoustic pressure on the oscillation dynamics of acoustic cavitation bubbles using high-speed shadowgraphy imaging of selected cavitation zones. They found that the number of bubbles grows when the negative pressure increases during the actuation cycle. However, no statistical evaluation of the bubble size distribution was done with respect to the actuation amplitude, phase and the boundary distance (gap) of sonotrode tip and opposite solid wall (see Figure 2).

The present study aims to gain a better understanding of the influence of the boundary conditions on the number and size distribution of cavitation bubbles created by an ultrasonic horn, hence on referred to as "bubble spectrum". For this purpose experiments are performed for different actuation amplitudes, phases and gap widths between the actuator tip and the opposite solid wall.

\section{Set-up and measurements}

The experimental set-up used for analyzing the bubble spectrum consists of an ultrasonic horn (sonotrode, Hielscher $\mathrm{GmbH}$ ) with a triggering unit, an octogonal water beaker and a shadowgraphy imaging system. The sonotrode is a dynamic oscillator with a working frequency of $20 \mathrm{kHz}$ and peak to peak Amplitude $\left(\mathrm{A}_{\mathrm{pp}}\right)$ that can be adjusted from $25 \mu \mathrm{m}$ to $50 \mu \mathrm{m}$. Recordings of the bubble clouds are obtained via shadowgraphy imaging for decreasing spacing between the sonotrode tip and the opposite solid wall. The shadowgraphy imaging system includes a $\mathrm{Nd}$ :YAG dual cavity laser (Quantel, Evergreen) for illumination, a double frame CCD-camera (Flowmaster, LaVision) and an optical diffusion light unit to achieve a homogeneous scattered light illumination. An illustration of the set-up is shown in Figure 2.

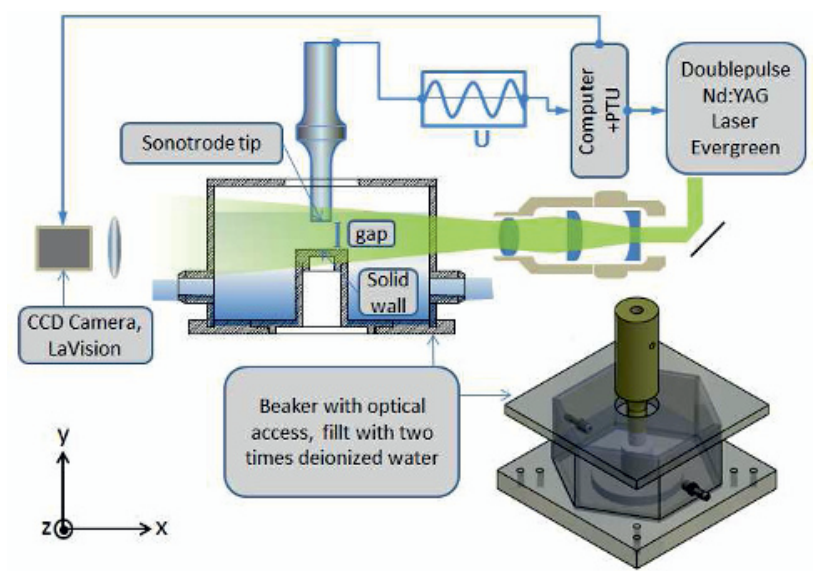

Figure 2. Test set-up for measuring the bubble spectrum

Deionized water was chosen as working fluid for the experiments. The water was stored for four days under constant room temperature and atmospheric pressure conditions. Previous to each measurement sequence the beaker is filled with 1.6 liter of water. Then the sonotrode is actuated for 10 minutes assuming an approximately constant level of dissolved gas is reached before the measurements. An octagonal shape of the beaker was used for good optical access. The cylindrically shaped sonotrode tip is positioned on the centerline axis of the beaker and reaches $5 \mathrm{~mm}$ deep into the water. The sonotrode tip is located above the opposite solid wall at two different distances of $17 \mathrm{~mm}$ and $5 \mathrm{~mm}$, respectively. This wall can be substituted by a viewing window for future investigations to obtain a bottom view of the twophase flow below the sonotrode during actuation. The pulsed dual cavity laser serves as light source providing exposure times of 6 nanoseconds. This allows to obtain sharp images even of highly dynamic cavitation bubbles. Recorded images span a field of view (FOV) of $22.13 \times 18.27 \mathrm{~mm}^{2}$, reaching an optical resolution of $8.91 \mu \mathrm{m}$ per pixel. Recordings are taken on the center plane below the sonotrode in the xy-plane (see Figure 2). The measurement sequences are synchronized with the actuation phase of the sonotrode, i. e. phase-locked images at 24 equidistant locations throughout the actuation phase are taken. In the following, bubble spectra at two different actuation phases, amplitudes (25 $\mu \mathrm{m}$ and $50 \mu \mathrm{m})$ and gap widths $(17 \mathrm{~mm}$ and $5 \mathrm{~mm})$ are compared.

\section{Measurement results and data analysis}

Figure 3a-d show representative shadowgraphy images of the cavitation zone under the sonotrode tip at $50 \mu \mathrm{m}$ and $25 \mu \mathrm{m}$ actuation amplitude, for $17 \mathrm{~mm}$ and $5 \mathrm{~mm}$ gap widths, respectively.

At $50 \mu \mathrm{m}$ actuation amplitude (Figure 3a, c) a typical cone bubble structure (CBS) is formed below the sonotrode tip, expanding, over the whole $17 \mathrm{~mm}$ gap width being partially deflected sideways at the viewing window surface. At $25 \mu \mathrm{m}$ actuation amplitude (Figure 3b, d) Acoustic Lichtenberg Figures (ALF) are increasingly formed, while the CBS is strongly reduced hardly reaching the lower surface wall. 


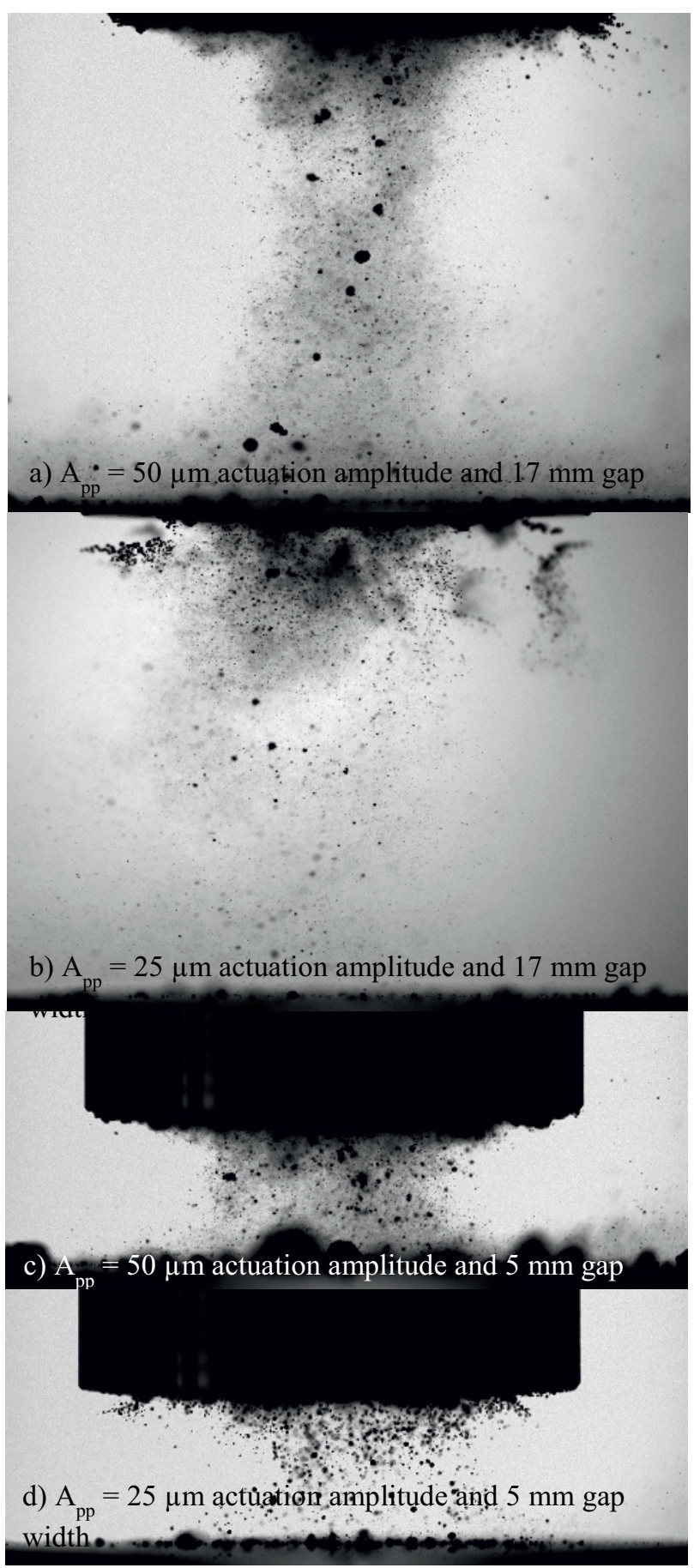

Figure 3. Snapshots of the bubble spectrum at $3 / 2 \pi$ of the actuation cycle (see Figure 7) for different actuation amplitudes and gap widths.

\subsection{Image Processing}

The total number and corresponding radii of cavitation bubbles were extracted from the shadowgraphy images with a self-written MATLAB algorithm. To detect the bubbles Hough-Transformation, Global-Thresholding, Watershed-Transformation, Prewitt Edge Detector, Roberts Edge Detector, Zero-Crossings Detector and the Canny Edge Detector [13] were tested and compared. However, the best detection results could be achieved with a self-written code based on an intensity gradient criterion for the bubbles edge detection. The following criteria were applied for image processing:

1. Time-averaged background images without actuation were subtracted from the bubble images of each recording sequence.

2. Gradient criterion: The gradient is manually set, representative for a grey value change across an in-focus bubble edge. Detected edges are denoted with a pixel value of one whereas all other pixels are given a zero value, resulting in a binarized image. Afterwards, all pixels inside a closed edge region are denoted with the value one.

3. Minimal pixel criterion: a detected region should be at least four pixels. Assuming a circular area this corresponds to an equivalent bubble radius of $10,05 \mu \mathrm{m}$.

4. Ratio criterion: The aspect ratio (width to height) of a region should not exceed 1.38.

A typical evaluation result after processing step 2 is shown in Figure 4 in which crosses mark initially detected regions.

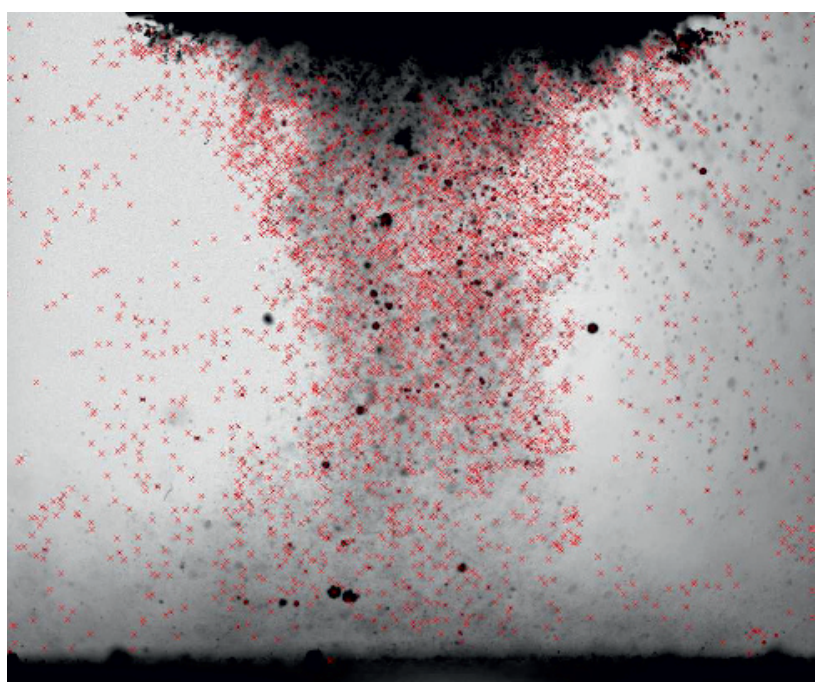

Figure 4. A typical evaluation results after processing step 2. Crosses mark the center of initially detected bubbles.

\subsection{Bubble spectra analyses}

\subsubsection{Rotational symmetry}

To ensure that the assumption of a time-averaged rotationally symmetric flow is valid, test measurements were carried out. For that purpose, an image series was taken at different z-positions below the sonotrode from the same measurement direction for region 1 and 2 (see Figure 5). The bubble frequency distribution was evaluated in two fields of interests of identical volume which are named region 1 and region 2 along the 
centerline $\mathrm{z}$ - and $\mathrm{x}$ - axis, respectively. Region 1 consists of the sum of four sub regions which refer to different imaging planes aligned along the measurement direction indicated in Figure 5.

The results of the comparison are shown in Figure 6. The amount of detected bubbles within the field of view (FOV) is indicated with $\mathrm{n}$ and the bubble radius with $\mathrm{r}$.

The curves are similar to each other and due to the fact that the standard deviations overlap, it can be concluded that the curves are the same within the given measurement uncertainty. Based on this results, it is assumed, that rotational symmetry is present.

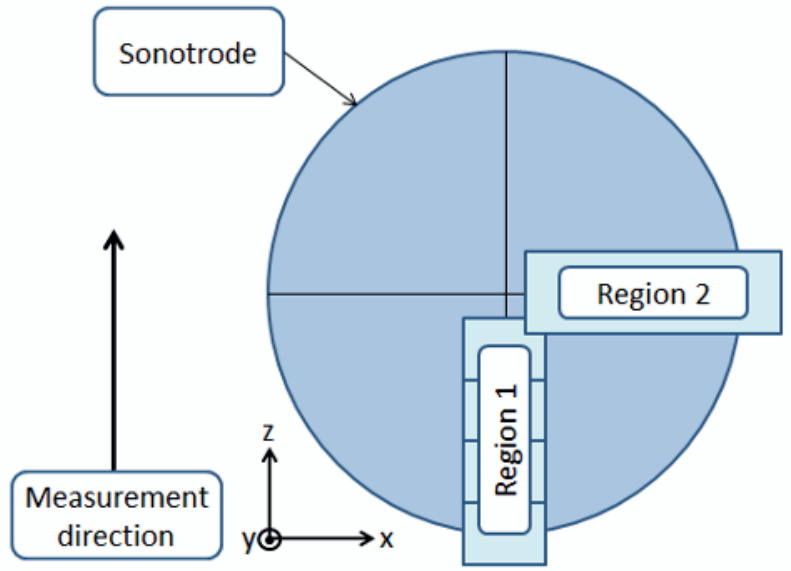

Figure 5. Illustration of the selected measurement volumes for the evaluation of rotational symmetry of the cavitation zone.

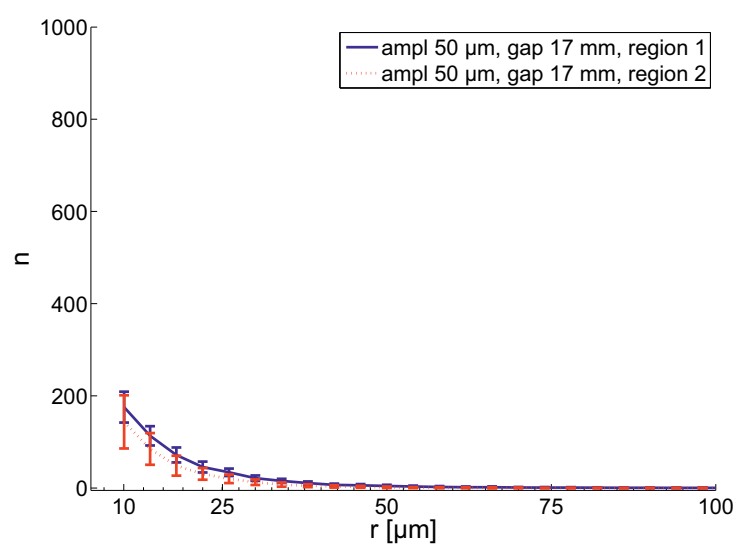

Figure 6. Comparison of the bubble spectra in $\mathrm{x}$ und $\mathrm{z}$ direction (compare Figure 5).

\subsubsection{The influence of the actuation phase}

To analyze the influence of the actuation phase on the bubble spectrum images are taken at different phases of the actuation. Figure 7 shows the measured sinusoidal actuation amplitude of the sonotrode tip of $50 \mu \mathrm{m}$. In all measurements 100 pictures were evaluated to determine the mean y-position of the sonotrode tip and its standard deviation as a function of the actuation phase $\alpha$.
The influence of the actuation phase on the bubble spectrum becomes evident in Figure 8 which shows two bubble spectra resulting from $50 \mu \mathrm{m}$ actuation amplitude and a $17 \mathrm{~mm}$ gap width. Measurement sequences were recorded at different actuation phases corresponding to $\alpha=\pi / 2$ and $\alpha=3 / 2 \pi$ (see Figure 7).

The two bubble spectra shown in Figure 8 show the largest relative deviations out of 20 phases being compared to each other. The largest number of bubbles was detected at an actuation phase of $\alpha=\pi / 2$, corresponding to a time instance of $5,5 \mu \mathrm{s}$ past the inversion of the direction of motion of the sonotrode. The actuation phase seems to influence the bubble spectrum, because at $\alpha=3 / 2 \pi$ the number of bubbles below a radius of approximately $50 \mu \mathrm{m}$ is increased in comparison to the amount of detected bubbles at $\alpha=\pi / 2$. Both functions show an exponential decrease with growing bubble radius.

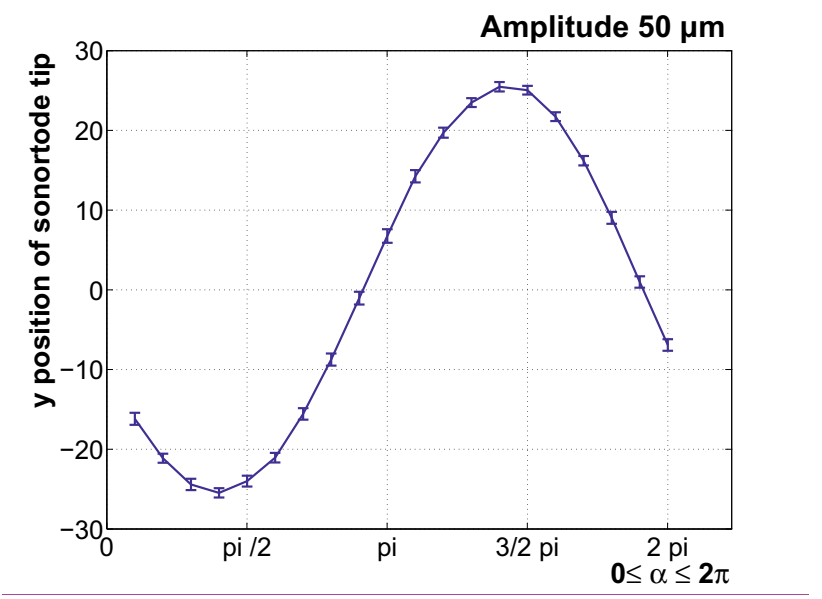

Figure 7. The y-position of the sonotrode tip is plotted as function of the actuation phase $\alpha$ with $0 \leq \alpha \leq 2 \pi$

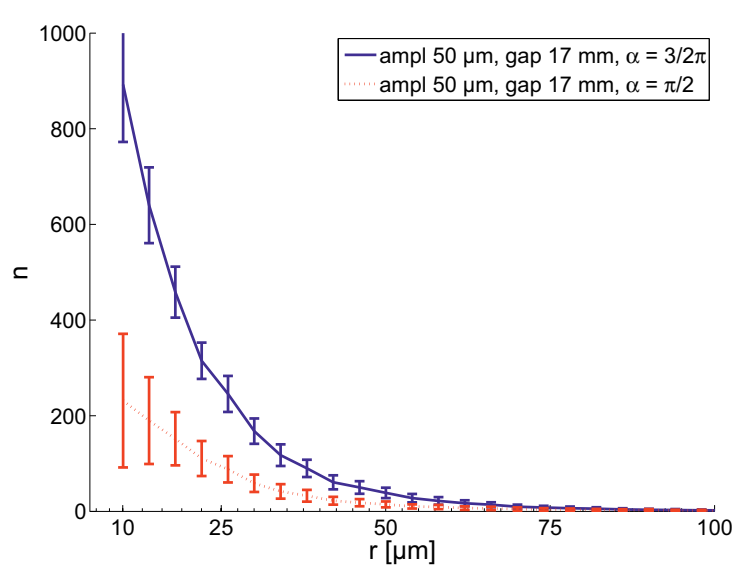

Figure 8. Bubble spectrum at $A_{p p} 50 \mu \mathrm{m}$ with a $17 \mathrm{~mm}$ gap at $\alpha=\pi / 2$ and $\alpha=3 / 2 \pi$ of the actuation phase.

In the following all results shown in this study were recorded at an actuation phase of $\alpha=3 / 2 \pi$. 


\subsubsection{The influence of the actuation amplitude}

Figure 9 shows the number of detected bubbles as function of the corresponding bubble radius for two different actuation amplitudes of $25 \mu \mathrm{m}$ and $50 \mu \mathrm{m}$ and at a $17 \mathrm{~mm}$ gap width between sonotrode tip and the solid wall.

The bubble spectrum resulting from an actuation amplitude of $50 \mu \mathrm{m}$ shows an exponential decrease in $n$ with growing radius (as shown in Figure 8). An increase in actuation amplitude leads to an increase especially of the small cavitation bubbles.

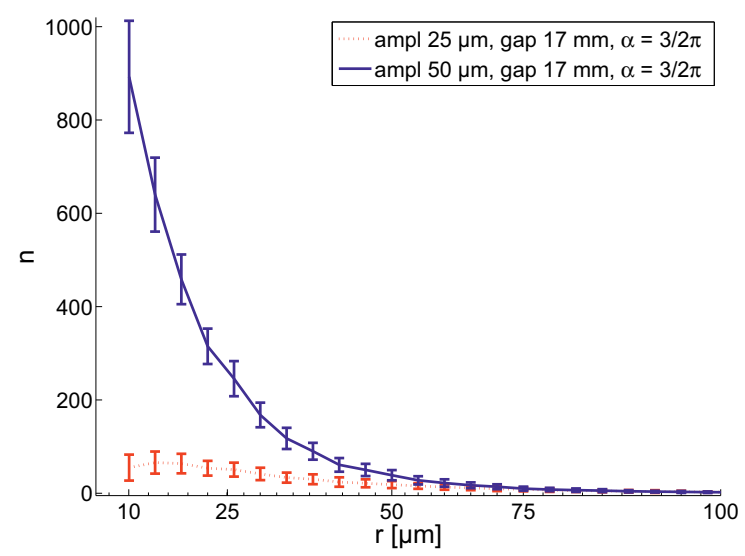

Figure 9. Bubble spectrum at $A_{p p} 50 \mu \mathrm{m}$ and $25 \mu \mathrm{m}$ with a 17 mm gap at $\alpha=3 / 2 \pi$.

Figure 10 shows the bubble spectrum at $50 \mu \mathrm{m}$ and 25 $\mu \mathrm{m}$ actuation amplitude for $5 \mathrm{~mm}$ spacing between sonotrode tip and the opposite solid wall.

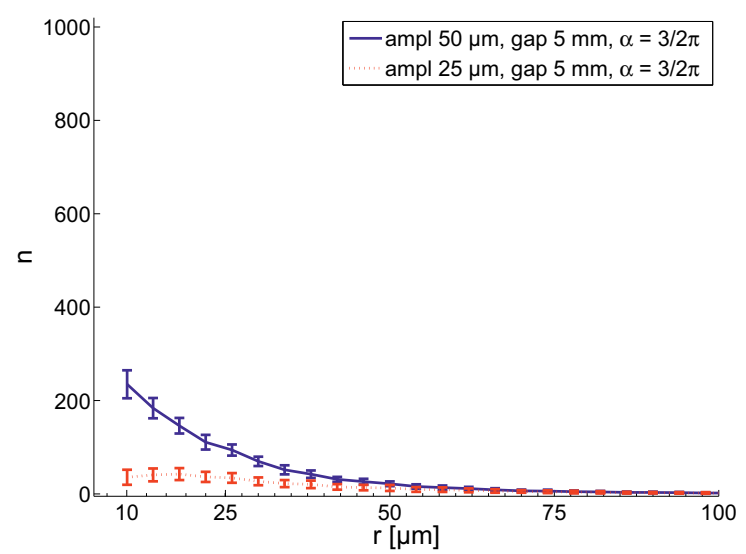

Figure 10. Bubble spectrum at $50 \mu \mathrm{m}$ and $25 \mu \mathrm{m}$ actuation amplitude for a $5 \mathrm{~mm}$ fluid gap at $\alpha=3 / 2 \pi$.

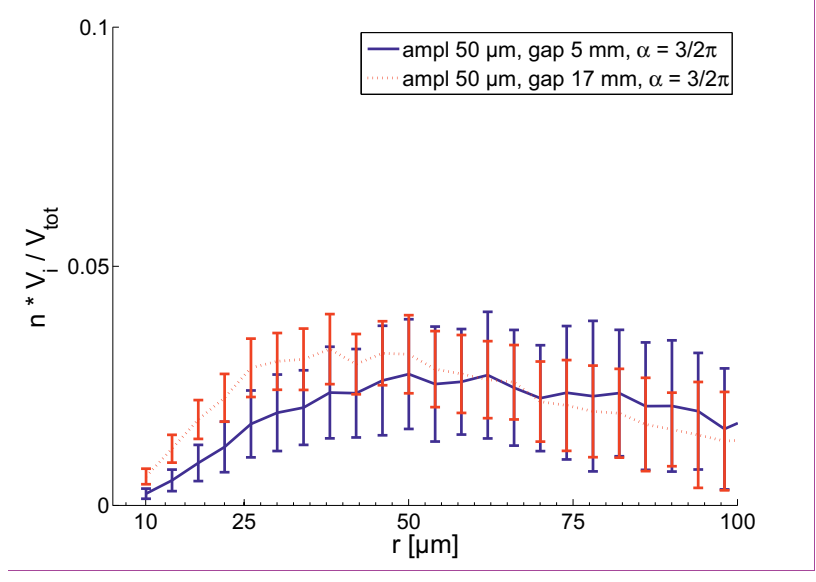

Figure 11. Comparison of the single bubble volumes to the total bubble volume at $A_{p p}=50 \mu \mathrm{m} \alpha=3 / 2 \pi . V_{i}$ is the volume of a single bubble, $V_{\text {tot }}$ is the volume of all accumulated bubbles.

The qualitative trend of the spectra is similar to those of Figure 9. However the overall amount of bubbles is reduced for both curves by a factor of about three.

Comparing the measurements results from Figure 9 and Figure 10 for an actuation amplitude of $50 \mu \mathrm{m}$ a clear difference in the number of detected bubbles is evident. However, comparing the cumulated bubble volume for bubbles of a specific radius $\left(\mathrm{n}^{*} \mathrm{~V}_{\mathrm{i}}\right)$ normalized by the total bubble volume $\left(\mathrm{V}_{\text {tot }}\right)$ no significant differences can be observed. This is shown in

Figure 11.

We therefore conclude that a different gap width between the sonotrode tip and the opposite solid wall has a minor effect on the bubble spectrum compared to the actuation phase and amplitude.

\section{Conclusion and future work}

In this investigation the influence of selected boundary conditions (actuation phase, amplitude and gap width) on the number and size distribution of cavitation bubbles was evaluated. An increase of the actuation amplitude leads to an increase in the number of small cavitation bubbles for $\mathrm{r} \leq 50 \mu \mathrm{m}$. A dependency between $\pi / 2$ and $3 / 2 \pi$ of the actuation phase could be determined: at $\alpha=$ $3 / 2 \pi$ a larger number of bubbles is detected than at $\alpha=$ $\pi / 2$. With a decreasing gap width between sonotrode and solid wall, the amount of bubbles decreases as well. The qualitative trend of the bubble spectra remains the same showing an exponential decrease in bubble number with growing bubble radius. However, a different gap width between the sonotrode tip and the opposite solid wall has a minor effect on the bubble spectrum compared to the actuation phase and amplitude.

In the next step the bubble spectrum in narrow gaps of less than $5 \mathrm{~mm}$ will be studied from a view from below the sonotrode front surface. For this purpose a viewing window will be implemented into the beaker instead of the opposing solid wall.

Additionally the distance of the bubbles to the sonotrode tip will be evaluated in order to assess the time 
integrated wall loads as function of the bubble wall distance [14]. This will allow to estimate the aggressiveness of the cavitation of single bubble collapses and thus the material damage in comparison to simulations performed by a companion paper [15]. Furthermore, bubble and fluid velocities will be analyzed to estimate the influence of the fluid flow on the bubble movement and distribution. High speed measurements will be performed to analyze sub harmonic oscillations, which could be observed in an in-house simulation [15]. After this, a parameter study will be carried out to analyze the influence of the beaker shape on the cavitation region which might be altered by wallreflected pressure waves propagating through the cavitation zone.

\section{References}

1. F. R. Young, Cavitation, World Scientific, (1989)

2. A. Brotchie, R. Mettin, F. Grieser, M. Ashokkumar, Phys. Chem. Chem. Phys., 11, 10029-10034 (2009)

3. D. G. Shchukin, E. Skorb, V. Belova, H. Möhwald, Adv. Mater. 23, 1922-1934 (2011)

4. J. W. Holl, J. Basic Eng., 92 (4), 681-688 (1970)

5. T. G. Leighton, A. J. Walton, M. J. W. Pickworth, Eur. Phys. J. E, 11, 47-50 (1989)

6. T. V. Anh, JUST, 73 B (2009)

7. A. Jayaprakesh, J.-K. Choi, G. L. Chahine, F. Martin, M. Donnelly, J.-P. Franc, Wear, 296, 619629 (2012)

8. V. A. Mayer, Annual Book of ASTM Standards Section 3, Material Test Methods and Analytical Procedures, 03.02, American Society for Testing and Materials (ASTM), 94-109 \& 558-571 (2010)

9. A. Moussatov, R. Mettin, C. Granger, T. Tervo, B. Dubus, W. Lauterborn, WCU Paris (2003)

10. T. Nowak, R. Mettin, W. Lauterborn, NAG/DAGA Rotterdam (2009)

11. W. Lauterborn, W. Hentschel, Cavitation Bubble, Ultrasonics 23 (3), 260 - 268 (1985)

12. H. A. Vaidya, Ö. Ertunç, A. Skupin, A. Delgado, GALA, 68, 1-7 (2013)

13. R.C. Gonzales, R. E. Woods, S. L. Eddings, Digital Image Processing Using MATLAB (Prentice Hall, 2004)

14. E.-A. Brujan, K. Nahen, P. Schmidt, A. Vogel, ISNA 15, 381-384 (2000)

15. S. Mottyll, S. Müller, J. Hussong, R. Skoda, Conference Proceedings Experimental Fluid Mechanics 13, submitted (2013) 